\title{
High expression of DDX20 enhances the proliferation and metastatic potential of prostate cancer cells through the NF-kB pathway
}

\author{
WEIGUO CHEN, PENG ZHOU and XIAOWEI LI \\ Department of Urology, Chengdu Military General Hospital, Chengdu, Sichuan 610083, P.R. China
}

Received February 18, 2015; Accepted April 7, 2016

DOI: $10.3892 /$ ijmm.2016.2575

\begin{abstract}
DEAD (Asp-Glu-Ala-Asp) box polypeptide 20 (DDX20), a member of the DEAD box protein family, encodes an RNA helicase. Previous research has revealed that DDX20 may act as a tumor suppressor in hepatocellular carcinoma and as a tumor promoter in breast cancer. These conflicting reports prompted us to explore the role of DDX20 in prostate cancer $(\mathrm{PCa})$. To gain insight into the functions of DDX20 in PCa, we examined DDX20 expression patterns in a PCa tissue microarray with $99 \mathrm{PCa}$ tissue samples. The results of immunohistochemical staining revealed that DDX20 expression is frequently upregulated in PCa tissues compared with that in the adjacent tissues and further clinicopathological analysis showed that the expression level of DDX20 closely correlates with tumor size, TNM stage (positive correlation) and patient prognosis (negative correlation). Both gain-of- and loss-of-function assays were performed in vitro; the overexpression of DDX20 enhanced the proliferation and metastatic potential of cancer cells and this was examined by performing a cell counting kit-8 (CCK-8) assay, wound healing assay and Transwell migration assay. Furthermore, we found that there is a positive correlation between the expression of matrix metallopeptidase 9 (MMP9) and DDX20 expression. These findings led us to examine whether DDX20 may exert effects through the NF- $\kappa B$ pathway. Luciferase reporter assays suggested that DDX20 altered the activity of NF- $\kappa \mathrm{B}$. Taken together, these findings show that DDX20 may promote the progression of $\mathrm{PCa}$ through the NF- $\mathrm{kB}$ pathway.
\end{abstract}

\section{Introduction}

Prostate cancer (PCa) is the second most frequently diagnosed cancer in Europe (1) and the second leading cause of cancer mortality in males in the USA (2). Although a complete

Correspondence to: Dr Weiguo Chen, Department of Urology, Chengdu Military General Hospital, 270 Rongdu Avenue, Chengdu, Sichuan 610083, P.R. China

E-mail: weiguochencd@163.com

Key words: prostate cancer, DDX20, proliferation, metastasis, MMP9, NF- $\mathrm{B}$ pathway understanding of the causes of PCa remains elusive, obesity, advancing age and family history have been established as the principal risk factors (3). Genetic alterations may also increase the risk of developing PCa, as suggested by associations with specific gene variants. Mutations in BRCA1 and BRCA2 (4), hereditary PCa gene 1, the androgen receptor and the vitamin D receptor (5), as well as fusion between TMPRSS2 and ETS family members, have also been implicated in $\mathrm{PCa}$ (6). Despite recent advances, the molecular mechanisms involved in the development and progression of $\mathrm{PCa}$ remain unclear.

DEAD (Asp-Glu-Ala-Asp) box polypeptide 20 (DDX20), a member of the DEAD box protein family, encodes an RNA helicase. Recently, the roles of DDX20 have been reported in hepatocellular carcinoma (HCC) (7) and breast cancer (8). In HCC, a deficiency of DDX20 was demonstrated to impair the functioning of miRNA-140 and lead to hepatocarcinogenesis. However, in breast cancer, DDX20 was identified as a biomarker and an oncogenic driver of metastasis. These contradictory findings prompted us to explore the role of DDX20 in PCa.

In the present study, we demonstrated that the expression of DDX20 is frequently increased in PCa. As DDX20 is reportedly associated with the metastasis of breast cancer, we examined whether DDX20 affects the same biological activities in PCa. The results of the present study indicate that DDX20 plays a similar role in PCa.

\section{Materials and methods}

Patients and sample collection. Twenty-four patients with $\mathrm{PCa}$, who underwent surgical resection at Chengdu Military General Hospital (Chengdu, China) between 2008 and 2014, were enrolled into the present study. All samples were frozen in liquid nitrogen immediately following surgical resection and stored at $-80^{\circ} \mathrm{C}$ until RNA extraction.

Ethics statement. All fresh tumor tissues and matched adjacent tissues were collected from patients with pathologically and clinically confirmed PCa. All human tumor tissues were obtained with written informed consent from patients. The Institutional Review Board of Chengdu Military General Hospital approved the use of the tumor sample in this study.

Immunohistochemistry (IHC). A PCa tissue microarray (TMA; HPro-Ade180PG-01) containing tissues from 99 cases (81 paired 
carcinoma and adjacent tissues as well as 18 cancer specimens) was purchased from Outdo Biotech (Shanghai, China). Staining of the TMA was performed according to standard IHC protocols. Following deparaffinization and dehydration of the TMA, endogenous peroxidase activity was blocked with $0.3 \%$ hydrogen peroxide (Sangon, Shanghai, China) at $37^{\circ} \mathrm{C}$ for $30 \mathrm{~min}$, and then antigen retrieval was performed by boiling in $10 \mathrm{mM}$ citrate buffer ( $\mathrm{pH}$ 6.0) for $15 \mathrm{~min}$. The sections were blocked with $10 \%$ bovine serum albumin (BSA; Sangon) for $1 \mathrm{~h}$ and incubated with DDX20 antibody (1:1,000; 11324-1-AP) and matrix metallopeptidase 9 (MMP9) antibody (1:1,000; 10375-2-AP) (both from ProteinTech, Chicago, IL, USA) overnight at $4{ }^{\circ} \mathrm{C}$. The next day, the TMA was incubated with horseradish peroxidase (HRP)-labeled anti-mouse secondary antibody (1:200; Dako, Carpinteria, CA, USA) for $1 \mathrm{~h}$ at room temperature. Antibody binding was detected using 3,3'-diaminobenzidine (DAB) in substrate chromogen solution (Dako). The TMA was counterstained with hematoxylin (Beyotime, Nantong, China) prior to dehydration and mounting. The final expression level of DDX20 was designated as a low and high expression group: score 0-1, low expression; 2-3, high expression. DDX20 expression was quantified by two independent pathologists. The slides were visualized using a Primostar FL2 microscope (Carl Zeiss, Oberkochen, Germany).

Cell culture. The PCa cell lines LNCaP, PC-3 and DU145 were obtained from the American Type Culture Collection (ATCC; Manassas, VA, USA). All of the cell lines were maintained in Dulbecco's modified Eagle's medium (DMEM) supplemented with $10 \%$ fetal bovine serum (FBS), $100 \mathrm{IU} / \mathrm{ml}$ penicillin and $100 \mu \mathrm{g} / \mathrm{ml}$ streptomycin (all from Gibco, Carlsbad, CA, USA) at $37^{\circ} \mathrm{C}$ in a $5 \%$ humidified $\mathrm{CO}_{2}$ atmosphere.

Reverse transcription-quantitative polymerase chain reaction (RT-qPCR). The surgical specimens were homogenized using a Mixer Mill MM 300 homogenizer (Qiagen, Chatsworth, CA, USA). Total RNA from these tissues and the PCa cell lines was then isolated using TRIzol reagent (Takara Bio, Inc., Otsu, Japan) and reverse transcribed using the PrimeScript RT-PCR kit (Takara Bio,Inc.) according to the manufacturer's instructions. Target gene expression was determined by performing qPCR with a SYBR Premix Ex Taq kit (Takara Bio, Inc.) and an ABI 7500 real-time PCR system (Applied Biosystems, Foster City, CA, USA). The primers for qPCR were designed as follows: DDX20 forward, 5'-CCGGGGAGAGGAAGAAA ATA-3' and reverse, 5'-ACTTCCACATCCCAATCCAC-3'; MMP9 forward, 5'-ACGACGTCTTCCAGTACCGA-3' and reverse, 5'-GCACTGCAGGATGTCATAGG-3'; and GAPDH forward, 5'-GGAGCGAGATCCCTCCAAAAT-3' and reverse, 5'-GGCTGTTGTCATACTTCTCATGG-3'. GAPDH was amplified as an internal control. We performed the assays according to the following program: $95^{\circ} \mathrm{C}$ for $30 \mathrm{sec}$, followed by 40 cycles at $95^{\circ} \mathrm{C}$ for $5 \mathrm{sec}$ and $60^{\circ} \mathrm{C}$ for $31 \mathrm{sec}$, and finally $95^{\circ} \mathrm{C}$ for $15 \mathrm{sec}, 60^{\circ} \mathrm{C}$ for $1 \mathrm{~min}$ and $95^{\circ} \mathrm{C}$ for $15 \mathrm{sec}$. The relative expression of DDX20 and MMP9 was calculated using the $2^{-\triangle \Delta C T}$ method with $18 \mathrm{~S}$ RNA as the control.

DDX20 RNA interference (RNAi). Specific small interfering RNAs (siRNAs; GenePharma, Shanghai, China) were designed to silence DDX20 and for the purpose of avoiding off-target effects, we used an siRNA pool which contained three siRNAs. The DU145 and the PC-3 cells were seeded at at a density of $30 \times 10^{4}$ cells in a final volume of $1.5 \mathrm{ml}$ in 6 -well plates. Pooled siRNAs were transfected at a final concentration of $10 \mathrm{nM}$ with Lipofectamine 2000 (Invitrogen, Carlsbad, CA, USA). The sequences for the DDX20 siRNAs were as follows: siNC, 5'-UUCUCCGAACGUGUCACGUTT-3'; si-1, 5'-TCTTTA TTCTTGATGAA-3'; si-2, 5'-GTGGATGATCGTATTT-3' and si-3, 5'-GTATTACAAAGTTGTCAA-3'.

Ectopic expression of DDX20. The expression plasmid containing the open reading frame of DDX20 and empty vector was purchased from GeneCopoeia (Guangzhou, China). The LNCaP cells $\left(30 \times 10^{4}\right.$ cells) were seeded in a 6 -well plate and transfected with $2 \mu \mathrm{g}$ plasmid using Lipofectamine 2000 (Invitrogen). After a $48 \mathrm{~h}$ incubation, stably transfected cells were selected using $1 \mathrm{mg} / \mathrm{ml} \mathrm{G} 418$ (Gibco) in DMEM and grown for 2 weeks. The G418-resistant colonies were isolated by a limited dilution approach. They were expanded and then maintained in regular growth medium containing $1 \mathrm{mg} / \mathrm{ml} \mathrm{G} 418$.

Western blot analysis. The total protein was extracted using RIPA lysis buffer (Beyotime, Haimen, China) according to the manufacturer's instructions and $50 \mu \mathrm{g}$ protein was separated by reducing SDS-PAGE, and transferred onto a nitrocellulose membrane. The membrane was then blocked in TBS buffer containing 5\% BSA (Sangon) for $1 \mathrm{~h}$. The membrane was incubated with primary antibodies for DDX20 (1:1,000; 11324-1-AP); MMP9 (1:1,000; 10375-2-AP), and GAPDH (1:5,000; 10494-1-AP, all from ProteinTech) overnight, and then followed by HRP-linked secondary antibody (\#7074; Cell Signaling Technology, Danvers, MA, USA). Immobilon ${ }^{\mathrm{TM}}$ Western Chemiluminescent HRP Substrate kit (Millipore Corp, Darmstadt, Germany) was used for detection.

Cell counting kit-8 (CCK-8) assay of cell viability. To evaluate changes in cell viability, CCK-8 assays were performed. The transfected cancer cells were seeded at a density of $4 \times 10^{4}$ cells/well in 96-well plates at a final volume of $100 \mu \mathrm{l}$ medium/well. Cell viability was quantified by adding $10 \mu \mathrm{l} \mathrm{CCK-8} \mathrm{(Dojindo,} \mathrm{Kumamoto,} \mathrm{Japan).} \mathrm{After} \mathrm{a} 1.5 \mathrm{~h}$ incubation, the plates were monitored at specific time points using a PowerWave XS Microplate reader (BioTek, Winooski, VT, USA), which measured absorbance at $450 \mathrm{~nm}$.

Wound healing assay. The transfected cancer cells were seeded onto 12-well plates and cultured until confluent. Wounds were generated using a sterile $200 \mu 1$ pipette tip (Axygen, Union City, CA, USA). The cells were then cultured for an additional $72 \mathrm{~h}$. Wound closure was assessed using an IX71 inverted microscope (Olympus Corp., Tokyo, Japan). The cell migration distance was measured using Adobe Illustrator CS5 software and compared with baseline measurements. Each experiment was performed in triplicate.

Invasion assay. For the Transwell migration assay, the transfected cells $\left(4 \times 10^{4}\right)$ were placed in the top chamber of each insert chamber (Millipore Corp.) with the Matrigel-coated membrane. The cells were trypsinized and resuspended in serum-free DMEM and $700 \mu \mathrm{l}$ complete medium was injected into the 

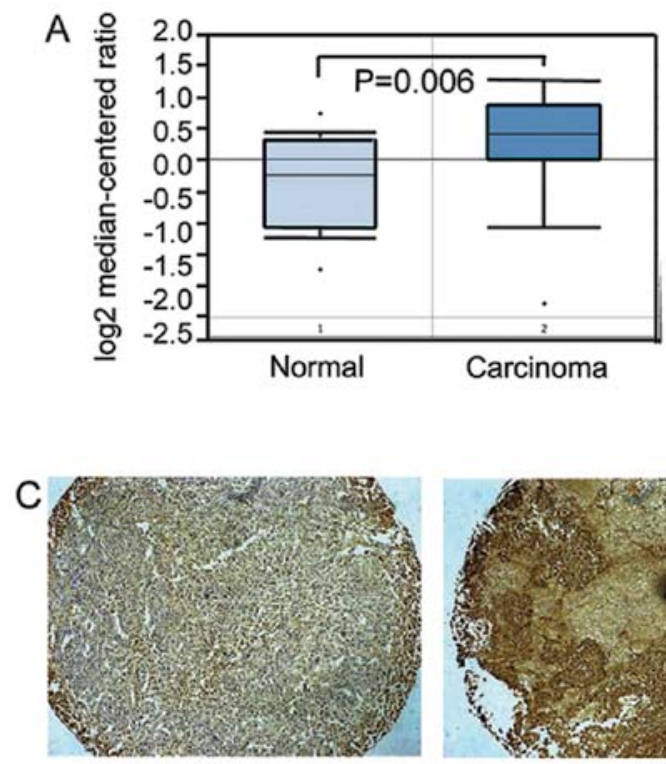

Normal

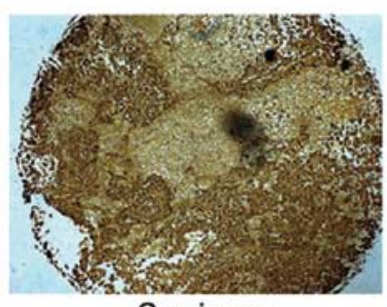

Carcinoma
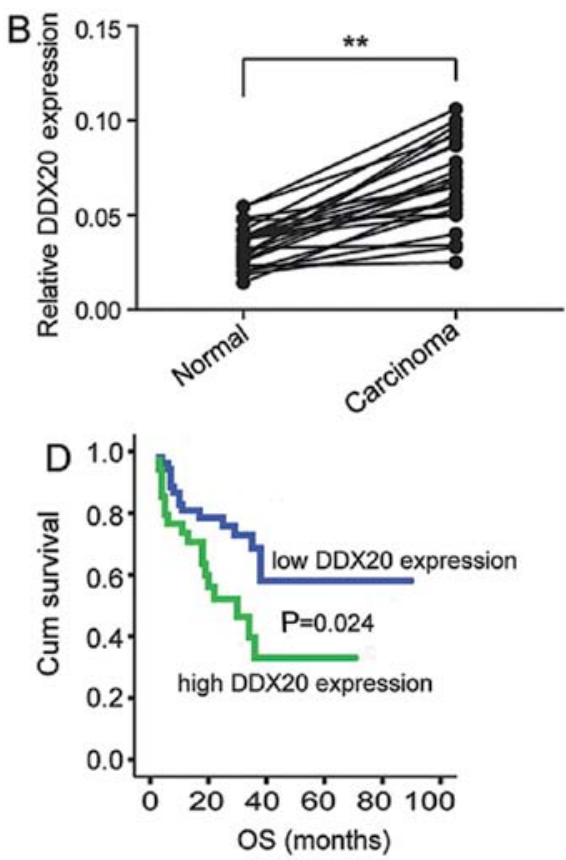

Figure 1. DDX20 expression is significantly upregulated in prostate cancer (PCa) compared with that in adjacent tissues and is closely associated with the prognosis of PCa. (A) Box plots derived from DDX20 expression data in the Oncomine database. Compared with normal prostate glands (left plot), the expression of DDX20 is significantly elevated in PCa $(\mathrm{P}=0.006)$. (B) The mRNA expression of DDX20 is significantly increased in fresh PCa tissues resected from 24 patients; ${ }^{* * *} \mathrm{P}<0.01$. (C) Representative images of DDX20 expression in PCa and adjacent tissues; magnification, x50. (D) Kaplan-Meier analysis of overall survival (OS) in groups of patients with either low or high expression of DDX20 (P=0.024). Cum, cumulative.

lower chamber. The plates were incubated for 48-72 $\mathrm{h}$, and then the medium and the cells remaining in the top chambers were removed. After fixation and staining with $0.1 \%$ crystal violet (Beyotime, Nantong, China), the cells that had migrated to the lower membrane of the inserts were counted and images were captured under an IX71 inverted microscope (Olympus Corp.).

Luciferase reporter assays. To perform luciferase reporter assays, the transfected cells were seeded in 96-well plates and transfected with a mixture of $100 \mathrm{ng} \mathrm{NF- \kappa B}$ reporter plasmids and 10 ng Renilla (Promega, Madison, WI, USA) according to the manufacturer's instructions for the Lipofectamine 2000 transfection system. Following $48 \mathrm{~h}$ of incubation, firefly and Renilla luciferase activities were measured sequentially in the cell lysates using the Dual-Luciferase Reporter Assay system (Promega).

Statistical analysis. Statistical analysis was conducted using SPSS 16.0 software (SPSS, Inc., Chicago, IL, USA). We performed Chi-square tests using cross-tabulation analysis in order to assess the relationships between the expression levels of DDX20 and clinicopathological factors. Overall survival (OS) was calculated using the Kaplan-Meier method. The survival distributions were compared through the log-rank test. The Student's t-test was used for comparisons between groups. A P-value $<0.05$ was considered to indicate a statistically significant difference.

\section{Results}

Expression of DDX20 is significantly upregulated in PCa. Recently, a study showed that DDX20 was highly expressed in human breast cancer and is associated with tumor metastasis (8). This prompted us to explore the functions of DDX20 in PCa. A
Table I. Correlation between DDX20 expression and key clinicopathological features.

\begin{tabular}{lccc}
\hline & \multicolumn{3}{c}{ DDX20 (n=99) } \\
\cline { 2 - 4 } Variable & Low & High & P-value \\
\hline Age (years) & & & \\
$\quad \leq 50$ & 34 & 13 & 0.183 \\
$>50$ & 31 & 21 & \\
Tumor size (cm) & & & $0.014^{\mathrm{a}}$ \\
$\leq 5$ & 28 & 28 & \\
$>5$ & 11 & 32 & $0.041^{\mathrm{a}}$ \\
Local infiltration & & & \\
Yes & 3 & 63 & $0.018^{\mathrm{a}}$ \\
No & 6 & 27 & \\
Gleason grade & & & \\
I & 2 & 40 & \\
II-III & 10 & 42 & \\
IV-V & 4 & &
\end{tabular}

${ }^{\mathrm{a}} \mathrm{P}<0.05$.

query of the Oncomine database revealed that notably DDX20 gene expression is significantly upregulated in PCa tissue compared with that in normal prostate gland tissue (Fig. 1A). To further delineate the expression profile of DDX20 in fresh $\mathrm{PCa}$ and adjacent tissues, we examined the mRNA levels of DDX20 in 24 paired adjacent and malignant tissues. As shown in Fig. 1B, the mRNA expression of DDX20 was significantly increased in the carcinomas compared with that in the adjacent 


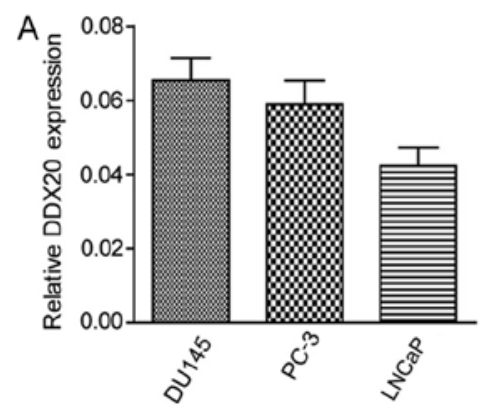

B

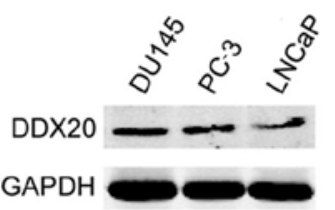

Figure 2. Levels of DDX20 in prostate cancer (PCa) cell lines. (A) Relative mRNA expression of DDX20 in three cell lines, with GAPDH as the internal control. (B) DDX20 protein expression was detected in three PCa cell lines by western blot analysis. GAPDH was used as a loading control.

tissues. Furthermore, we detected DDX20 in a TMA which contained 99 malignant tissues and 81 non-malignant adjacent tissues. We found that DDX20 was more commonly detected in the malignant tissues, as shown in Fig. $1 \mathrm{C}$ and this was indicated by stronger DDX20 staining in the carcinoma tissues than in the adjacent tissues. We then analyzed the correlation between DDX20 expression and patient prognosis and found that the patients with high DDX20 expression had lower OS $(\mathrm{P}=0.024)$ (Fig. 1D). The analysis of the correlation between DDX20 expression and key clinicopathological features is presented in Table I and shows that DDX20 expression is associated with tumor size, local infiltration and Gleason grade.

Human PCa cell lines express DDX20. The significant correlation between DDX20 expression and clinicopathological features prompted us to investigate the effect of DDX20 on various cellular functions during PCa development. Firstly, we examined the expression of DDX20 using RT-qPCR; the DU145 and PC-3 cells exhibited higher expression levels of DDX20 compared with the LNCaP cells (Fig. 2A). To confirm the protein expression of DDX20 in the three cell lines, we performed western blot analysis and the results showed the same pattern as the RT-qPCR results (Fig. 2B).

DDX20 contributes to the proliferation, migration and invasiveness of human PCa cells. We selected the DU145 and PC-3 cell lines for further RNAi analysis and the LNCaP cell line for use in overexpression experiments in order to examine DDX20 expression. The transfection of the DU145 and PC-3 cells with
A

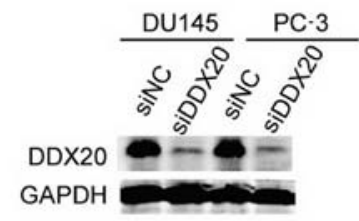

C

siNC
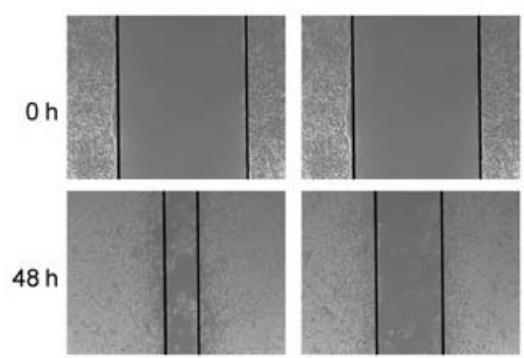

E

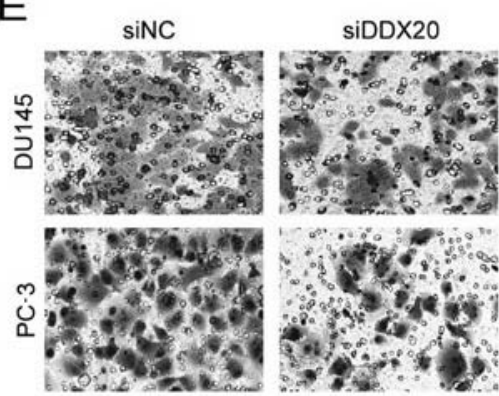

B

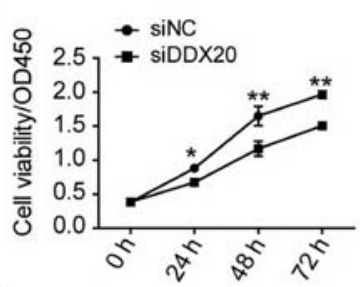

D

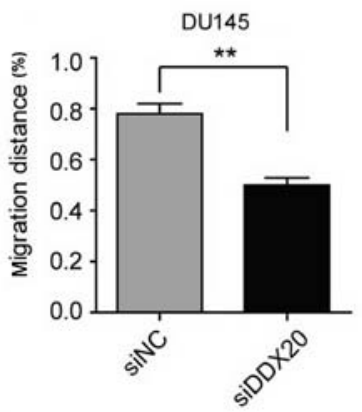

F

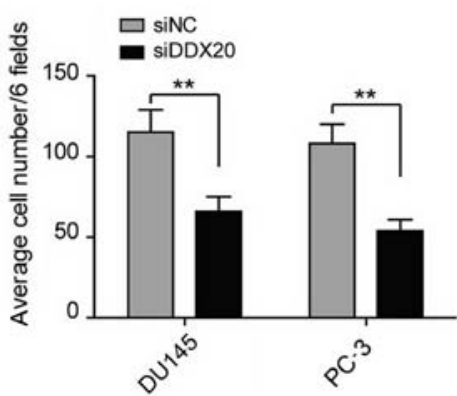

Figure 3. Silencing DDX20 inhibits the proliferation and metastatic potential of prostate cancer (PCa) cell lines. (A) DDX20 expression levels were detected in DU145 and PC3 cells $48 \mathrm{~h}$ after transfection with siRNA targeting DDX20. (B) Effect of DDX20 knockdown on the cell viability of DU145 cells as measured by a cell counting kit-8 (CCK-8) assay (values are the means \pm SD). (C and D) Analysis of cell migration by performing wound-healing assays. Statistical data are expressed as the means \pm SD. (E and F) Cell invasion was analyzed by performing the Matrigel-coated Transwell invasion assay (magnification, $x 200$ ). Statistical data are expressed as the means $\pm \mathrm{SD}$. All data are representative of three independent experiments. ${ }^{*} \mathrm{P}<0.05$; ${ }^{* *} \mathrm{P}<0.01$. 
A

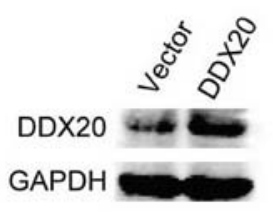

C
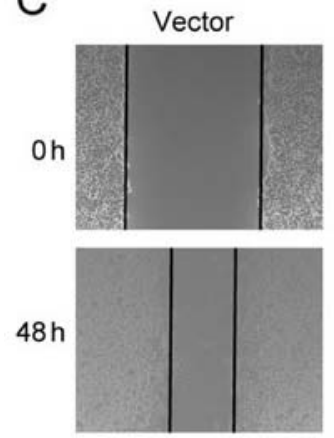

E

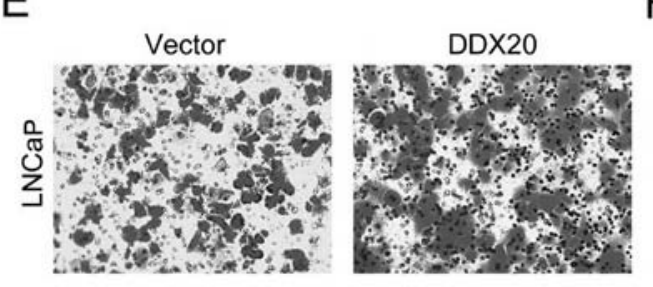

B

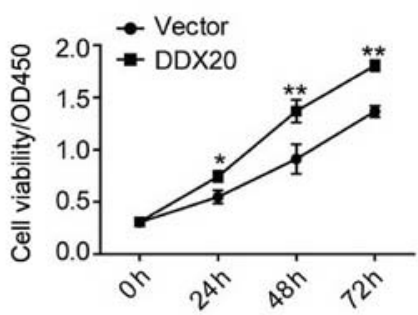

D

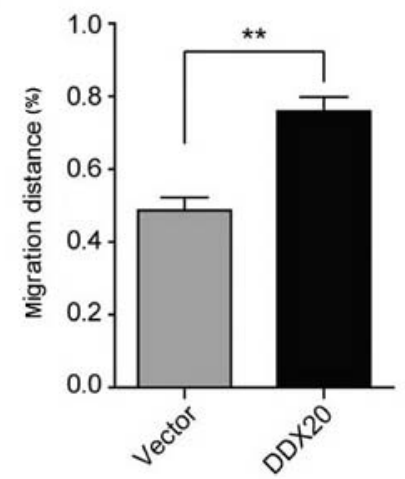

F

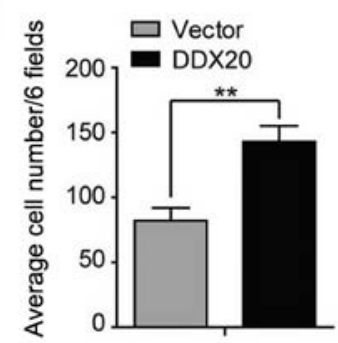

Figure 4. Ectopic expression of DDX20 enhances the proliferation and metastatic potential of LNCaP cells. (A) DDX20 levels were detected in LNCaP cells following transfection with the plasmid inducing the overexpression of DDX20. (B) Cell viability analysis of LNCaP cells when DDX20 was elevated, as measured by CCK-8 assay (values are the means \pm SD). (C and D) Analysis of cell migration by performing wound-healing assays. Statistical data are expressed as the means \pm SD. (E and F) Cell invasion was analyzed by performing the Matrigel-coated Transwell invasion assay (magnification, $x 200$ ). Statistical data are expressed as the means $\pm \mathrm{SD}$. All data are representative of three independent experiments. ${ }^{*} \mathrm{P}<0.05 ;{ }^{* *} \mathrm{P}<0.01$.

DDX20 siRNA reduced DDX20 expression (Fig. 3A). We studied various cellular functions, namely proliferation, migration and invasion, following siRNA treatment. To examine cell proliferation, we performed CCK-8 cell viability assays and found that cell viability decreased significantly in the DDX20silenced cells (Fig. 3B). We then assessed cell migratory behavior. After silencing DDX20, we performed wound-healing assays and found that DDX20 depletion led to decreased migration (Fig. 3C and D). Given that the suppression of DDX20 affects cell motility, we next explored the effects of decreasing DDX20 expression on the invasiveness of the malignant cells by performing the Matrigel-coated Transwell invasion assay. In the DU145 and PC-3 cells, knockdown of DDX20 resulted in $40-50 \%$ fewer cells invading through the Matrigel-coated inserts (Fig. 3E and F). Taken together, these results demonstrate that DDX20 depletion led to the inhibition of cell proliferation, migration and invasion.

Ectopic expression of DDX20 contributes to cell proliferation, migration and invasiveness. To further substantiate our findings, we then evaluated whether the ectopic expression of DDX20 had the potential to adversely affect LNCaP cells. We found that the ectopic expression of DDX20 markedly elevated the expression of DDX20 (Fig. 4A). The ectopic expression of DDX20 increased LNcaP cell viability (Fig. 4B). In addition, the ectopic expression of DDX20 in the LNCaP cells promoted cell motility and enhanced the invasive capacity of the LNCaP cells (Fig. 4C-F). Taken together, both loss-of- and gain-of-function experiments demonstrate that DDX20 expression concurrently affects the proliferation, migration and invasiveness of PCa cells.

DDX20 expression correlates with MMP9 levels in $\mathrm{PCa}$ tissues. Taking into account the promigratory effect of DDX20 in PCa cell lines and the associated mechanisms reported in other types of cancer, we decided to analyze the expression of MMP9, which has been reported to play a pivotal role in the DDX20-metastasis axis (8), in the same cohorts used in Fig. 1B. Similarly to DDX20, we observed higher mRNA expression levels of MMP9 in the PCa tissues compared with those in the adjacent tissues (Fig. 5A) and there was a positive correlation between DDX20 and MMP9 mRNA levels (Fig. 5B). To further substantiate this finding, we performed immunohistochemical analysis to examine the expression of MMP9 in the same cohort used in Fig. 1C. Notably, similarly to DDX20, the group of PCa patients with high expression of MMP9 were more likely to have a poor prognosis $(\mathrm{P}=0.036)(\mathrm{Fig} .5 \mathrm{D})$ and 
A

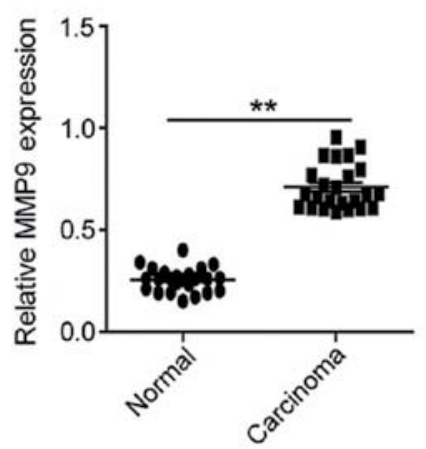

C

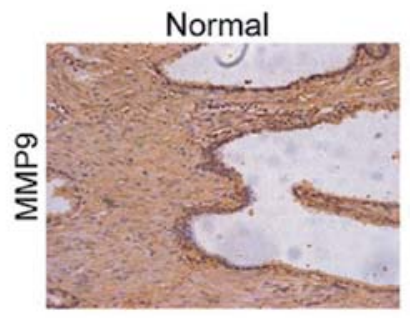

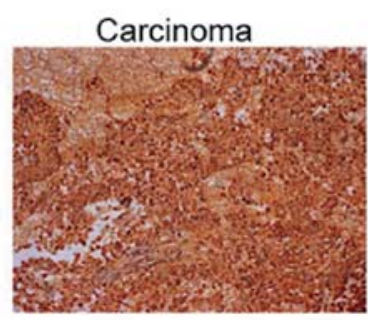

B

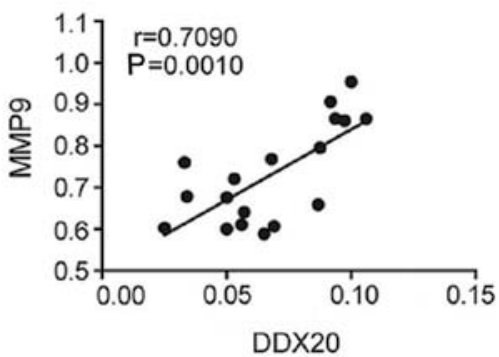

$\mathrm{D}$

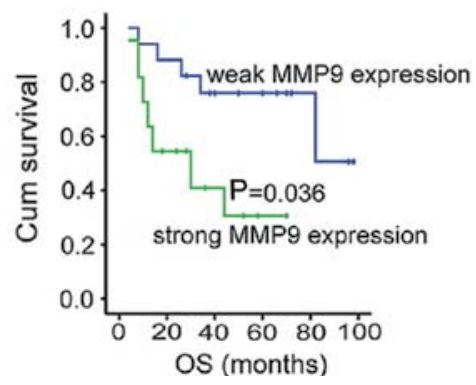

Figure 5. Matrix metallopeptidase 9 (MMP9) expression correlates with DDX20 expression in prostate carcinoma (PCa) tissues. (A) The mRNA expression of MMP9 is elevated in PCa tissues compared with that in the adjacent tissues (values are the means \pm SD). (B) Correlation analysis of the relative mRNA levels of DDX20 and MMP9. (C) Representative images of MMP9 expression in PCa (right panel) and adjacent tissues (left panel). (D) Kaplan-Meier analysis of overall survival $(\mathrm{OS})$ in groups of patients with either high or low expression of MMP9 $(\mathrm{P}=0.036)$. ${ }^{* *} \mathrm{P}<0.01$. Cum, cumulative.

A

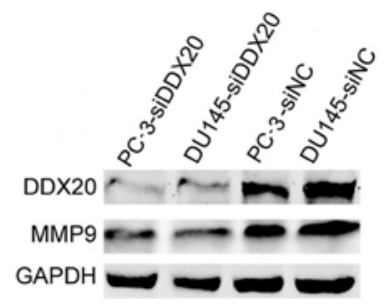

C

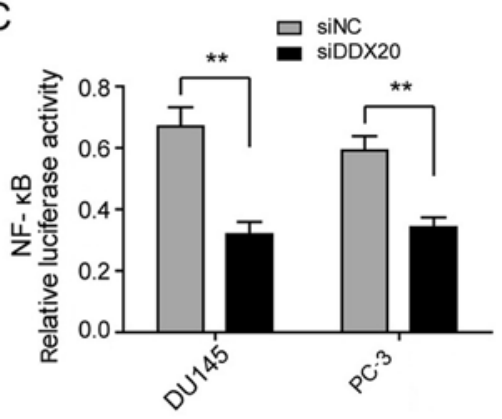

B

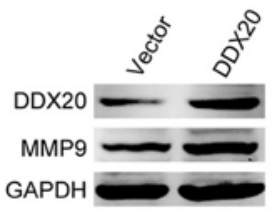

D

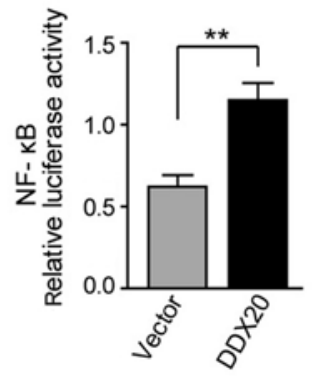

Figure 6. DDX20 regulates matrix metallopeptidase-9 (MMP9) expression through the NF-kB pathway. (A and B) The expression of DDX20 and MMP9 were examined following transfection. (C and D) Representative dual-luciferase reporter assays showed that DDX20 affects NF- $\kappa B$ signaling. Data are representative of three independent experiments. ${ }^{* *} \mathrm{P}<0.01$.

similar clinicopathological features were also obtained from the same TMA (Table II) and the same clinicopathological features significantly correlated with MMP9 expression. Taken together, the findings of the present study indicate that DDX20 may also exert effects in PCa through MMP9.

$D D X 20$ regulates $N F-\kappa B$ signaling in $P C a$ cell lines. Given the strong correlation between DDX20 and MMP9 in

Table II. Correlation between MMP9 expression and key clinicopathological features.

\begin{tabular}{lccc}
\hline & \multicolumn{3}{c}{ MMP9 (n=99) } \\
\cline { 2 - 4 } Variable & Low & High & P-value \\
\hline Age (years) & & & \\
$\quad \leq 50$ & 24 & 23 & 0.294 \\
$>50$ & 32 & 20 & \\
Tumor size (cm) & & & \\
$\quad 55$ & 36 & 20 & $0.026^{\mathrm{a}}$ \\
$>5$ & 18 & 25 & \\
Local infiltration & & & $0.002^{\mathrm{a}}$ \\
Yes & 11 & 23 & \\
No & 42 & 23 & $0.040^{\mathrm{a}}$ \\
Gleason grade & & & \\
I & 2 & 1 & \\
II-III & 16 & 34 & \\
IV-V & 26 & 20 & \\
\hline
\end{tabular}

${ }^{\mathrm{a}} \mathrm{P}<0.05$. MMP9, matrix metallopeptidase 9 .

PCa tissues, we then examined whether silencing or ectopically expressing DDX20 would reduce or increase the levels of MMP9, respectively. We found that silencing DDX20 resulted in the inhibition of MMP9 protein levels in the DU145 and PC-3 cell lines (Fig. 6A). Conversely, the ectopic expression of DDX20 resulted in the increased expression of MMP9 in the LNCaP cell line (Fig. 6B). These findings suggest that DDX20 may regulate MMP9 expression in order 
to enhance the migration and invasiveness of PCa cells. As $\mathrm{NF}-\kappa \mathrm{B}$ is considered to be the major transcriptional regulator of MMP9 $(9,10)$, we then examined whether the alterations in MMP9 expression following changes in DDX20 levels, are due to NF- $\kappa \mathrm{B}$ activity. We performed luciferase reporter assays and the results are shown in Fig. $6 \mathrm{C}$ and $\mathrm{D}$; the suppression of DDX20 in the DU145 and PC-3 cells led to the downregulation of NF- $\kappa \mathrm{B}$ activity (Fig. 6C). By contrast, the overexpression of DDX20 elevated NF- $\kappa$ B activity (Fig. 6D). Taken together, our findings suggest that DDX20 promotes the migration and invasiveness of $\mathrm{PCa}$ cells by regulating the NF- $\mathrm{B}-\mathrm{MMP} 9$ axis.

\section{Discussion}

In the present study, we report that DDX20 is elevated in the majority of PCa tissue samples and the high expression of DDX20 negatively correlates with patient prognosis. In addition, we have demonstrated that PCa cells with high expression of DDX20 are more prone to be proliferative and invasive.

It has been previously reported that DDX20 increases MMP9 levels which are associated with metastasis and invasion in breast cancer through the activation of NF- $\mathrm{BB}(8)$. In the present study, a positive correlation between DDX20 and MMP9 expression was observed in both PCa tissues and cell lines. In addition, the findings of the luciferase reporter assays suggested that DDX20 also exerted promigratory effects through $\mathrm{NF}-\kappa \mathrm{B}$ signaling in PCa cells. Taken together, these results provide further evidence that DDX20 acts as an oncogenic protein in $\mathrm{PCa}$ as well as in breast cancer.

Notably, two other studies have reported that DDX20 acts as a tumor suppressor in HCC. Zender et al employed an oncogenomics-based in vivo RNAi screen that identified DDX20 as a potential tumor suppressor in liver cancer (11). Takata et al revealed that DDX20 suppresses NF- $\kappa$ B activity by regulating miRNA-140 function in order to inhibit the progression of liver cancer $(7,12)$. We are unable to explain the contradictory results observed in studies of DDX20 in different types of cancer. However, given that it exerts disparate effects in various types of cancer through the same pathway, namely the MMP9 and NF- $\kappa$ B signaling pathway, we suggest that the ways in which DDX20 regulates the expression of MMP9 and $\mathrm{NF}-\kappa \mathrm{B}$ are dependent on the type of cancer. As PCa and breast cancer are gender-related cancers, we suggest that there may be an association between the effects of DDX20 and sex hormones; however, further studies are warranted.

In conclusion, we have demonstrated that there is high expression of DDX20 in PCa tissues and it may be useful as a potential prognostic marker in $\mathrm{PCa}$. In addition, we have examined the biological functions of DDX20 in PCa cell lines. Furthermore, we have provided preliminary evidence for the molecular mechanisms responsible for the effects of DDX20.
The contradictory effects of DDX20 in different types of cancer suggest that it may play a key role in the development and progression of cancer and therefore be a potential therapeutic target.

\section{References}

1. Ferlay J, Autier P, Boniol M, Heanue M, Colombet M and Boyle P: Estimates of the cancer incidence and mortality in Europe in 2006. Ann Oncol 18: 581-592, 2007.

2. Siegel R, Naishadham D and Jemal A: Cancer statistics, 2012. CA Cancer J Clin 62: 10-29, 2012.

3. Hankey BF, Feuer EJ, Clegg LX, Hayes RB, Legler JM, Prorok PC, Ries LA, Merrill RM and Kaplan RS: Cancer surveillance series: interpreting trends in prostate cancer - part I: Evidence of the effects of screening in recent prostate cancer incidence, mortality, and survival rates. J Natl Cancer Inst 91: 1017-1024, 1999.

4. Struewing JP, Hartge P, Wacholder S, Baker SM, Berlin M, McAdams M, Timmerman MM, Brody LC and Tucker MA: The risk of cancer associated with specific mutations of BRCA1 and BRCA2 among Ashkenazi Jews. N Engl J Med 336: 1401-1408, 1997.

5. Gallagher RP and Fleshner N: Prostate cancer: 3. Individual risk factors. CMAJ 159: 807-813, 1998.

6. Beuzeboc P, Soulié M, Richaud P, Salomon L, Staerman F, Peyromaure M, Mongiat-Artus P, Cornud F, Paparel P, Davin JL and Molinié V: Fusion genes and prostate cancer. From discovery to prognosis and therapeutic perspectives. Prog Urol 19: 819-824, 2009 (In French).

7. Takata A, Otsuka M, Yoshikawa T, Kishikawa T, Hikiba Y, Obi S, Goto T, Kang YJ, Maeda S, Yoshida H, et al: MicroRNA-140 acts as a liver tumor suppressor by controlling NF- $\mathrm{B}$ activity by directly targeting DNA methyltransferase 1 (Dnmt1) expression. Hepatology 57: 162-170, 2013.

8. Shin EM, Hay HS, Lee MH, Goh JN, Tan TZ, Sen YP, Lim SW, Yousef EM, Ong HT, Thike AA, et al: DEAD-box helicase DP103 defines metastatic potential of human breast cancers. J Clin Invest 124: 3807-3824, 2014.

9. Chou YC, Sheu JR, Chung CL, Chen CY, Lin FL, Hsu MJ, Kuo YH and Hsiao G: Nuclear-targeted inhibition of NF-kappaB on MMP-9 production by N-2-(4-bromophenyl) ethyl caffeamide in human monocytic cells. Chem Biol Interact 184: 403-412, 2010.

10. Ricca A, Biroccio A, Del Bufalo D, Mackay AR, Santoni A and Cippitelli M: bcl-2 over-expression enhances NF-kappaB activity and induces mmp-9 transcription in human MCF7(ADR) breast-cancer cells. Int J Cancer 86: 188-196, 2000.

11. Zender L, Xue W, Zuber J, Semighini CP, Krasnitz A, Ma B, Zender P, Kubicka S, Luk JM, Schirmacher P, et al: An oncogenomics-based in vivo RNAi screen identifies tumor suppressors in liver cancer. Cell 135: 852-864, 2008.

12. Takata A, Otsuka M, Yoshikawa T, Kishikawa T, Kudo Y, Goto T, Yoshida $\mathrm{H}$ and Koike $\mathrm{K}$ : A miRNA machinery component DDX20 controls NF- $\kappa$ B via microRNA-140 function. Biochem Biophys Res Commun 420: 564-569, 2012. 